\title{
Order Static Filter and Histogram Equalization are in Pipe Line for Noise Removal
}

\author{
Ankita Agarwal \\ M.Tech Student \\ MIET \\ Meerut
}

\author{
Tarun Kumar \\ Assistant Professor \\ CSED, Vidya College of \\ Engineering, \\ Meerut
}

\author{
Vishal Jayaswal \\ Assistant Professor \\ Meerut Institute of Engineering \\ and Technology \\ Meerut
}

\begin{abstract}
Whenever an image is reconstructed, the quality of reconstructed image is calculated in terms of various quality parameters. MSE is considered as one of the most reliable and widely used quality parameter; however, we are using a new universal image quality index $\mathrm{Q}$, which proves to be better than MSE. An improvisation of the same has also been proposed in this paper.
\end{abstract}

\section{General Terms}

Types of noise, filtering, image de-noising.

\section{Keywords}

Noise reduction, order static filtering, histogram equalization, noises.

\section{INTRODUCTION}

An image may be defined as a two-dimensional function $\mathrm{f}(\mathrm{x}$, $y$ ), where $x$ and $y$ are spatial coordinates, and the amplitude of $f$ at any pair of coordinates $(x, y)$ is called the intensity or gray level of the image at that point. An image is called digital image if value of $\mathrm{x}, \mathrm{y}$ and $\mathrm{f}$ are all finite and discrete. [1]

Noise is the deviation in intensity value of $\mathrm{f}$ from the original value and is the consequence of error in image acquisition process thus leading to an image having pixel values that do not reveal the actual intensities of real image. Digital images are prone to a wide range of types of noise. It is fundamental requisite to eradicate noise from image maintaining its features unharmed thus making it a good quality image.

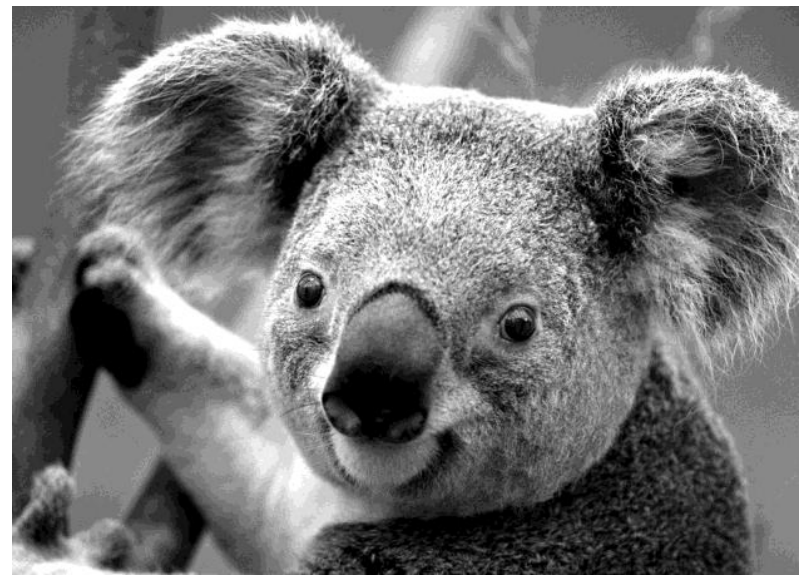

Fig 1: Input Image (koala.jpg)

\section{DIGITAL IMAGE NOISE MODEL}

The noise in an image is useless information. The dissimilarity is usually referred to as noise. Noise is a significant flaw in the image that can take numerous diverse forms and arises from a variety of sources such as heat generated might release electrons from the image sensors itself, as a consequence contaminating the "actual" photoelectrons. Noise is an interruption that affects a signal and may alter the information carried by the signal. Image noise can also instigate due to the electronic noise in the sensors in the digital cameras or scanner circuitry. Many types of noises exist today.

Noise can be classified into 3 types according to mixing with signal which are as follows.[2][11]

2.1 Additive noise- $f(x, y)$ is original image, $f^{\prime}(x, y)$ is noisy image and $\mathrm{n}(\mathrm{x}, \mathrm{y})$ is noise function which return random values. It is given by equation:

$f^{\prime}(x, y)=f(x, y)+n(x, y)$

Additive noise is autonomous of pixel values in original image. Gaussian noise is a type of additive noise. Gaussian noise is numerical noise. Gaussian noise and normal distribution (Gaussian distribution) both have identical probability density function (PDF). Additionally, the values that the noise can acquire are Gaussian-distributed.[3]

\section{Gaussian noise:}

$$
p(z)=\frac{1}{\sqrt{2 \pi} \sigma} e^{-(z-\mu)^{2} / 2 \sigma^{2}}
$$

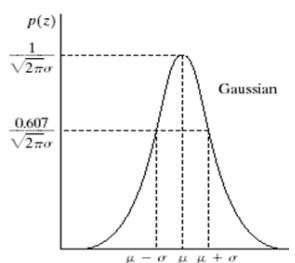

Fig 2: PDF of Gaussian noise

Gaussian noise is evenly distributed over the image $\mathrm{i}$ e each pixel in noisy image is summation of true pixel value and random Gaussian distributed noise value

Principal sources of Gaussian noise in digital images emerge during acquisition e.g. sensor noise caused by reduced lighting and/or elevated temperature, and/or transmission. In digital image processing, spatial filter can be used to reduce Gaussian noise, despite the fact that when an image is smoothed, an adverse effect may lead to the blurring of image edges and details. The median filter is sometimes not as instinctively good at dealing with large amounts of Gaussian 
noise as the mean filter.

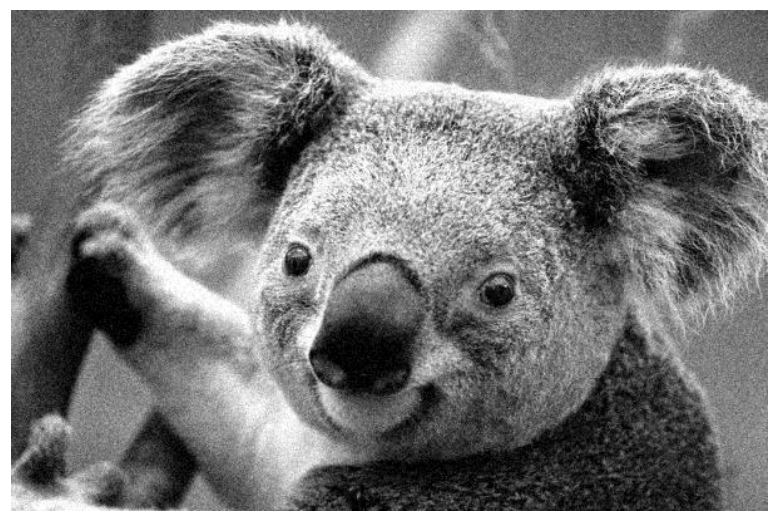

Fig 3: koala_gaussian_noise.jpg

2.2 Multiplicative noise- It is signal reliant form of noise whose magnitude is related to the value of original pixel

$$
\begin{aligned}
& f^{\prime}(x, y)=f(x, y)+n(x, y) * \\
& f(x, y) \ldots \ldots . . .(2)
\end{aligned}
$$

This kind of noise is also called speckled noise. This noise gives a magnified view of the original image. Radar waves can interfere constructively or destructively to produce light and dark pixels known as speckle noise. Speckle noise is commonly observed in radar sensing systems, although it may appear in any type of remotely sensed image utilizing coherent radiation.

Ideally, speckle noise in radar images must be completely removed. However, in practice it can be reduced significantly. It gives a grainy effect to the image. In general, speckle noise can be reduced by spatial filtering.[4][10]

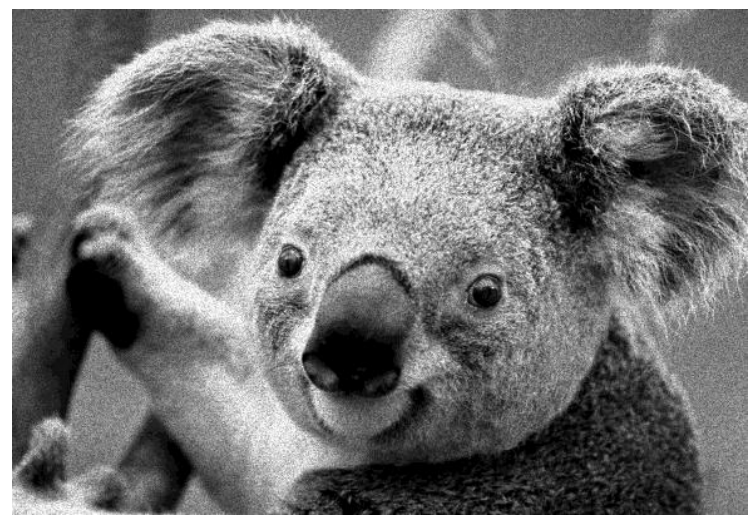

Fig 4: koala_spec_noise.jpg

2.3 Impulse Noise- It has property of either leaving a pixel unchanged with probability 1-p or replacing it in total with probability $\mathrm{p}$. Impulse noise is typically outcome of an inaccuracy in transmission or an atmospheric or man- made disruption. It is neither additive nor multiplicative in nature. This replaces pixel value by impulse

$$
f^{\prime}(x, y)=f(x, y) \cup n(x, y)
$$

This type of noise is at times called as salt-and pepper noise. This kind of noise is a noise typically seen on images. It represents itself as randomly occurring white and black pixels.
An image containing this type of noise will have dark pixels in light regions and light pixels in dark regions. This type of noise can be caused by analog-to-digital converter errors, bit errors during transmission, etc.[5]

$$
\text { Impulse noise: }
$$$$
p(z)=\left\{\begin{array}{lll}
p_{a} & \text { for } z=a \\
p_{b} & \text { for } z=b \\
0 & \text { otherwise }
\end{array}\right.
$$

Fig 5: PDF of Impulse Noise

An efficient noise reduction method for this type of noise is a median filter or a morphological filter. Given the probability $r$ (with $0 \leq \mathrm{r} \leq 1$ ) that a pixel is degraded, we can introduce saltand-pepper noise in an image by setting a fraction of $r / 2$ randomly selected pixels to black, and another fraction of $r / 2$ randomly selected pixels to white.

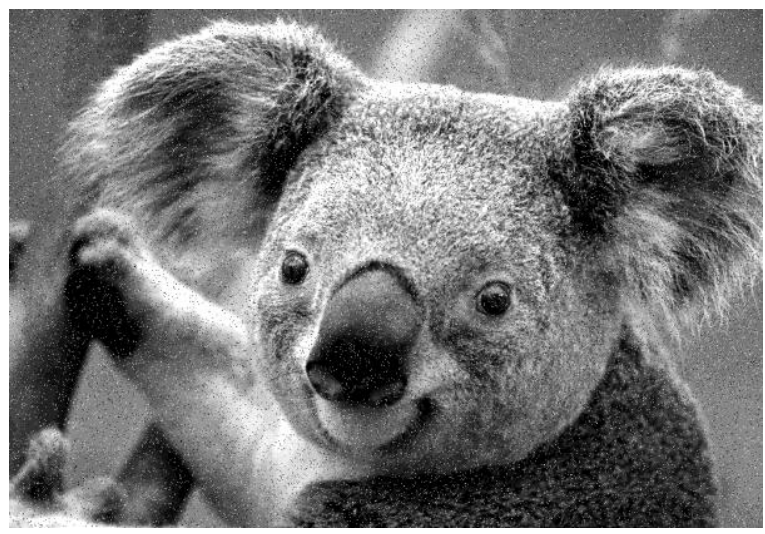

Fig 6: koala_snp_noise.jpg

\section{FILTERING TECHNIQUES}

Filtering in image processing is a core function that is used to accomplish noise reduction. It is a prevailing process. The preference of filter is determined by nature of task performed by filter and behavior and type of data. Filters are used to eliminate noise from digital image while keeping the information of image conserved.[6] [13]

Filtering techniques are furthur classified into following 2 types:

\section{Linear filters.}

2. Non- linear filters

\subsection{Linear Filters}

Linear filters are used to eradicate certain type of noise. Gaussian or Averaging filters are suitable for this purpose. These filters also tend to blur the sharp edges, destroy the lines and other fine details of image, and perform badly in the presence of signal dependent noise.

\subsection{Non- Linear Filters}

In recent years, a variety of non-linear median type filters have been developed to surmount the weakness of linear 
filter.Spatial filters that are based on ordering the pixel values that make up the neighbourhood operated on by the filter.[12][14]

Order-statistics filters are nonlinear spatial filters whose response is based on ordering (ranking) the pixels contained in the image area encompassed by the filter, and then replacing the value of the center pixel with the value determined by the ranking result[7][9]

\subsubsection{Max filter}

$$
\hat{f}(x, y)=\max _{(s, t) \in S_{x y}}\{g(s, t)\}
$$

\subsubsection{Min Filter}

$$
\hat{f}(x, y)=\min _{(s, t) \in S_{x y}}\{g(s, t)\}
$$

\subsection{Image Restoration process}

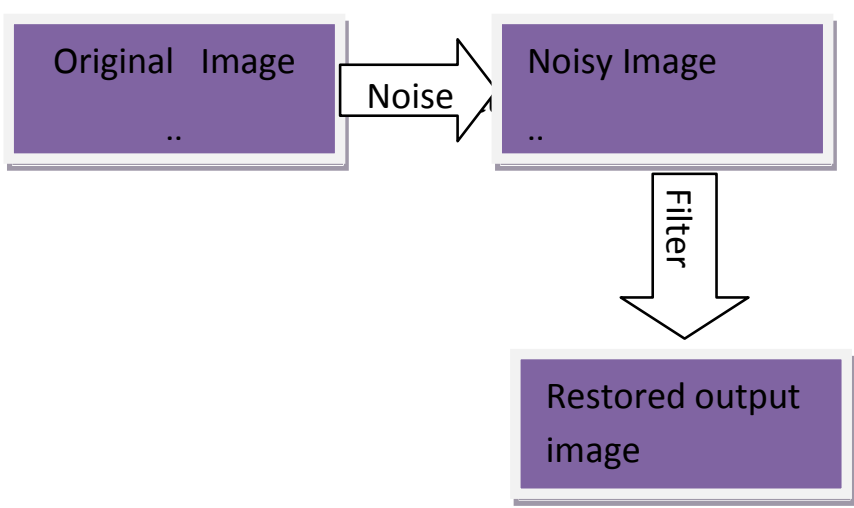

Fig 8: Image Restoration Process

\section{A UNIVERSAL IMAGE QUALITY INDEX}

It is a new worldwide objective image quality index which is easy to evaluate and relevant to variety of image processing applications. This anticipated index is calculated by modeling any image alteration as a blend of 3 factors: loss of correlation, luminance distortion, and contrast distortion, rather than using conventional error summation method. Even though the new index is precisely defined and no human visual system model is explicitly employed, experiments on different image distortion types indicates that it performs appreciably better than extensively used distortion metric mean square error.

let $\mathrm{x}=\left\{x_{i} \mid i=1, \ldots . N\right\}$ and $\mathrm{y}=\left\{y_{i} \mid i=1, \ldots . N\right\}$ be the original and test image signals respectively. The proposed quality index is defined as:

$$
\mathrm{Q}=\frac{4 \sigma_{x y} \overline{x y}}{\left(\sigma_{y}^{2}+\sigma_{x}^{2}\right)\left[\bar{x}^{2}+\bar{y}^{2}\right]}
$$

Where

$$
\bar{x}=\frac{1}{N} \sum_{N}^{1} x_{i}
$$

$$
\begin{gathered}
\bar{y}=\frac{1}{N} \sum_{N}^{1} y_{i} \\
\sigma_{x}^{2}=\frac{1}{N-1} \sum_{N}^{1}\left(x_{i}-\bar{x}\right)^{2} \\
\sigma_{x}^{2}=\frac{1}{N-1} \sum_{N}^{1}\left(x_{i}-\bar{x}\right)^{2} \\
\sigma_{x y}=\frac{1}{N-1} \sum_{N}^{1}\left(x_{i}-\bar{x}\right)\left(y_{i}-\bar{y}\right)
\end{gathered}
$$

The dynamic range of $\mathrm{Q}$ is $[-1,1]$. the best value 1 is

is achieved if and only if $y_{i}=x_{i}$ for all $\mathrm{i}=1,2 \ldots \ldots \ldots \mathrm{N}$. the lowest value of -1 occurs when $y_{i}=2 \bar{x}-x_{i}$ for all $\mathrm{i}=1,2 \ldots \ldots \mathrm{N}$. this quality index models any distortion as a combination of 3 different factors: loss of correlation, luminance distortion, and contrast distortion. In order to understand this, we rewrite $\mathrm{Q}$ as product of 3 components.

$$
Q=\frac{\sigma_{x y}}{\sigma_{x} \sigma_{y}} \cdot \frac{2 \bar{x} \bar{y}}{\left(\bar{x}^{2}\right)+\left(\bar{y}^{2}\right)} \cdot \frac{2 \sigma_{x} \sigma_{y}}{\sigma_{y}^{2}+\sigma_{x}^{2}}
$$

The first component is correlation coefficient between $\mathrm{x}$ and $\mathrm{y}$, which measures the linear correlation between $\mathrm{x}$ and $\mathrm{y}$ and its dynamic range is $[-1,1]$. The best value 1 is obtained when $y_{i}=a x_{i}+\mathrm{b}$ for all $\mathrm{i}=1,2 \ldots \ldots . \mathrm{N}$. when $\mathrm{a}$ and $\mathrm{b}$ are constants and $\mathrm{a}>0$. Even if $\mathrm{x}$ and $\mathrm{y}$ are linearly related, there still might be relative distortions between them, which are evaluated in the second and third component. The second component with a value range of $[0,1]$, measures how close the mean illuminance is between $\mathrm{x}$ and $\mathrm{y}$. it equals one if and only if $\bar{x}=\bar{y} . \sigma_{x}$ and $\sigma_{y}$ can be viewed as estimate of contrast of $\mathrm{x}$ and $\mathrm{y}$. so the third component measures how similar the contrasts of image are. Its range of values is also $[0,1]$, where the best value 1 is achieved if and only if $\sigma_{x}=\sigma_{y} \cdot[8]$

\section{RESULT}

\section{1 min filter application}

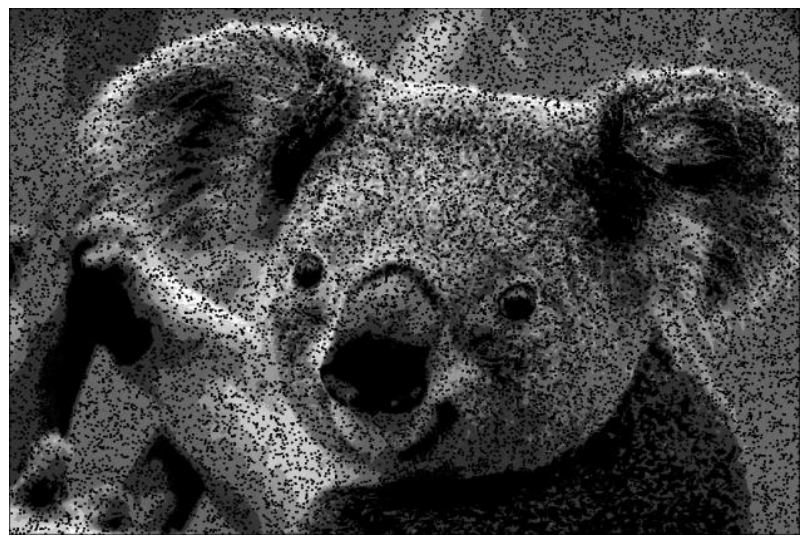

Fig 9: K_snp_min.gif 


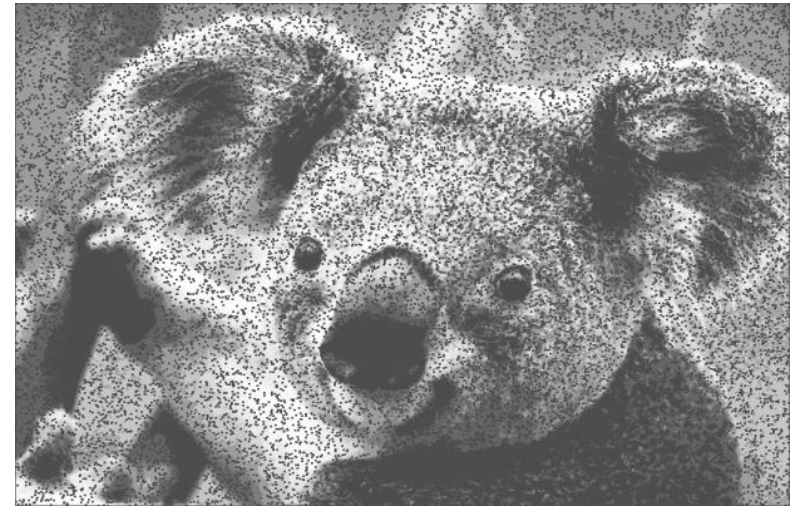

Fig 10: K_snp_min_hist.gif

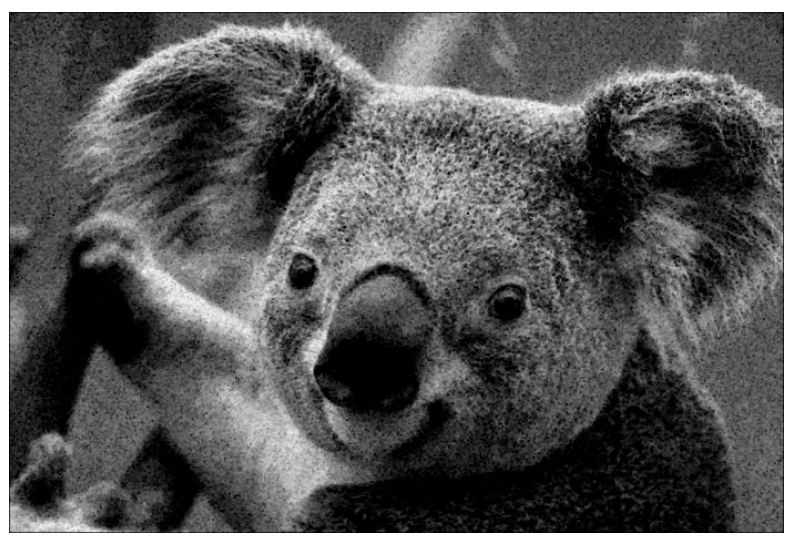

Fig 11: K_gauss_min.gif

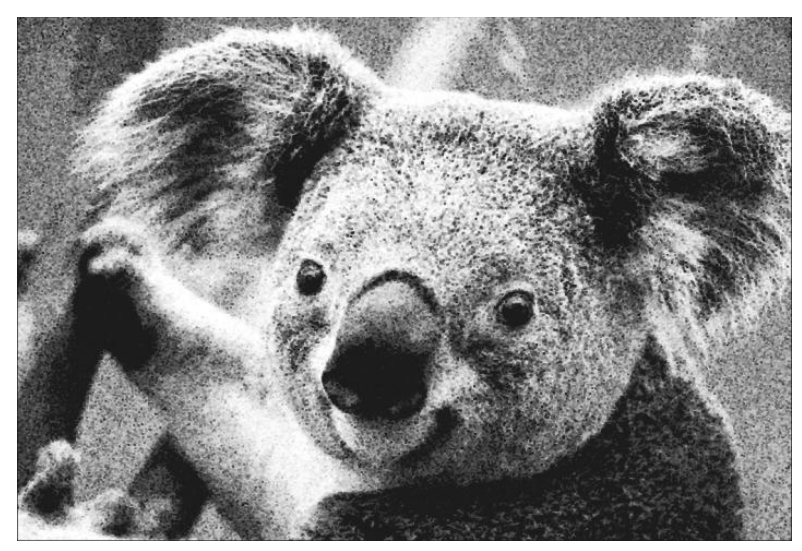

Fig 12: K_gauss_min_hist.gif

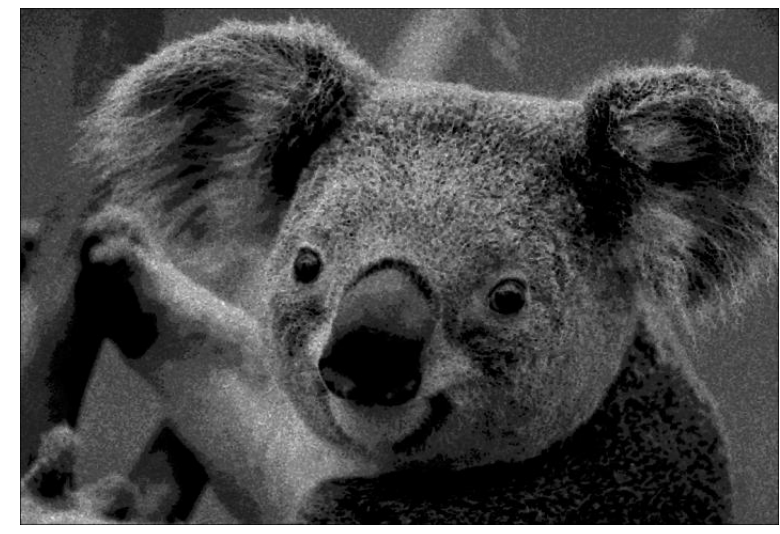

Fig 13: K_spec_min.gif

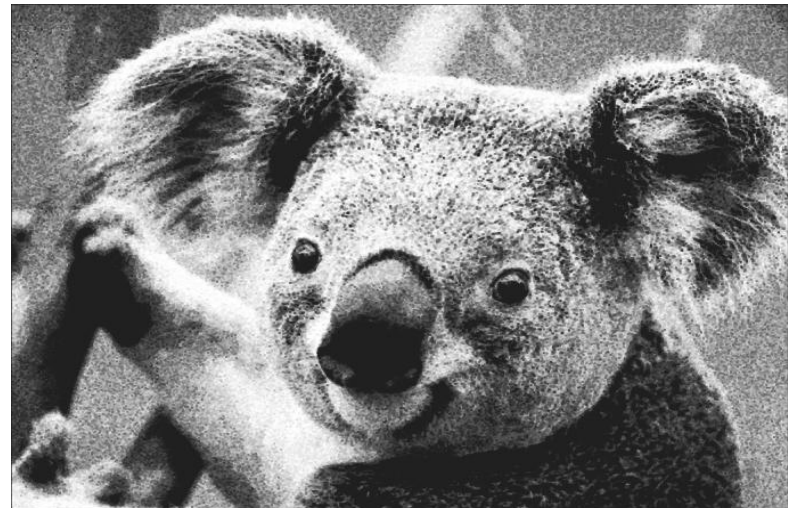

Fig 14: K_spec_min_hist.gif

Table 1. Value of new quality index

\begin{tabular}{|c|c|c|}
\hline \multirow{2}{*}{ Noise/ Technique } & \multicolumn{2}{|c|}{ New Quality Index } \\
\cline { 2 - 3 } & After Min filter & $\begin{array}{c}\text { After Min filter } \\
\text { and Histogram } \\
\text { equalization }\end{array}$ \\
\hline Salt and pepper & 0.6643 & 0.8562 \\
\hline Speckle & 0.6622 & 0.9425 \\
\hline Gaussian & 0.7210 & 0.9477 \\
\hline
\end{tabular}

\section{2 max filter application}

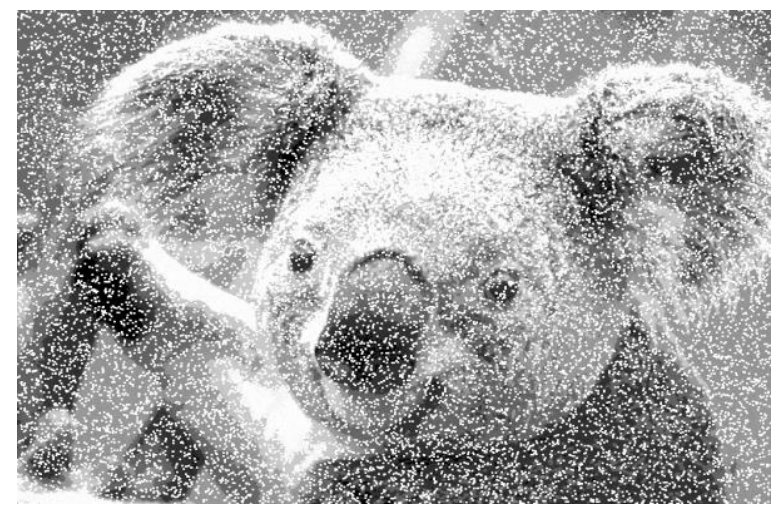

Fig 15: K_snp_max.gif

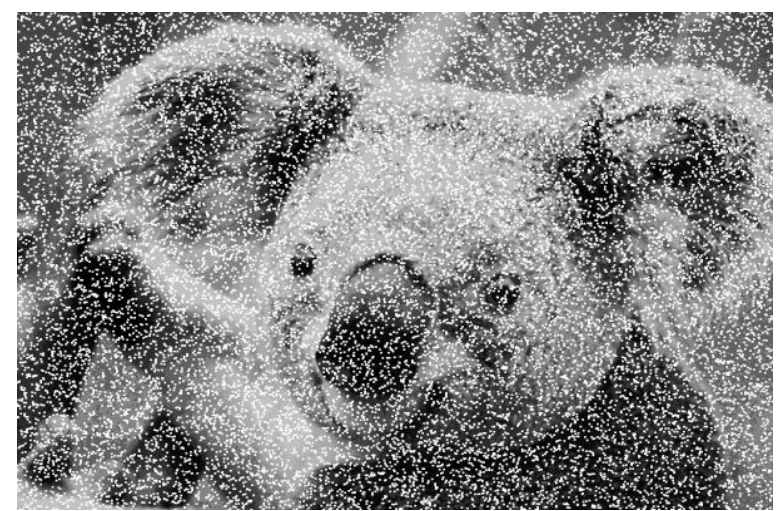

Fig 16: K_snp_max_hist.gif 


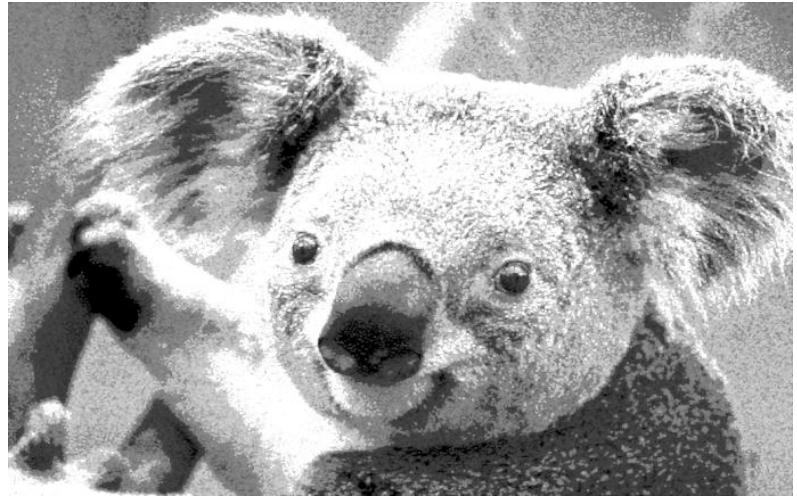

Fig 17: K_spec_max.gif

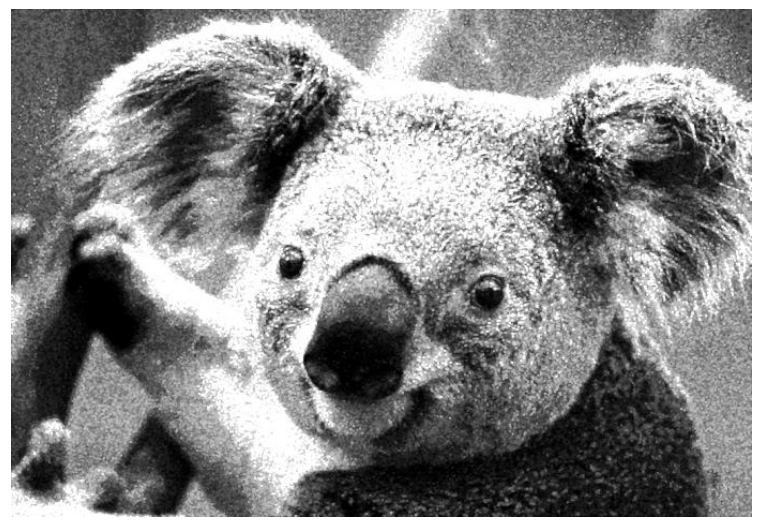

Fig 18: K_spec_max_hist.gif

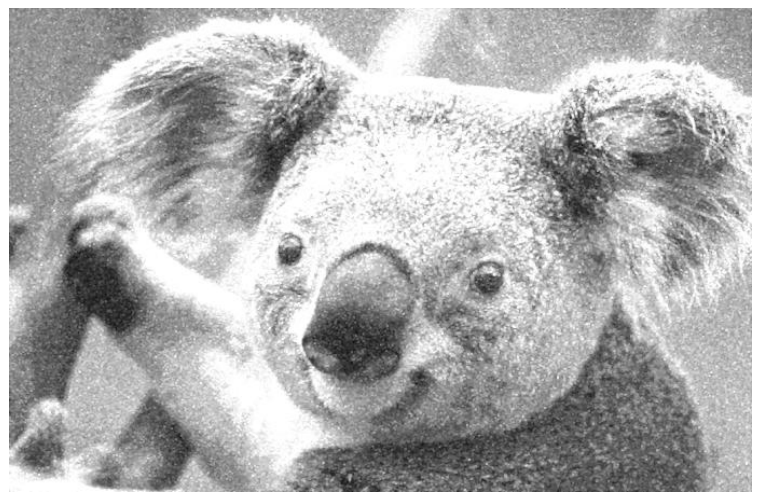

Fig 19: K_gauss_max.gif

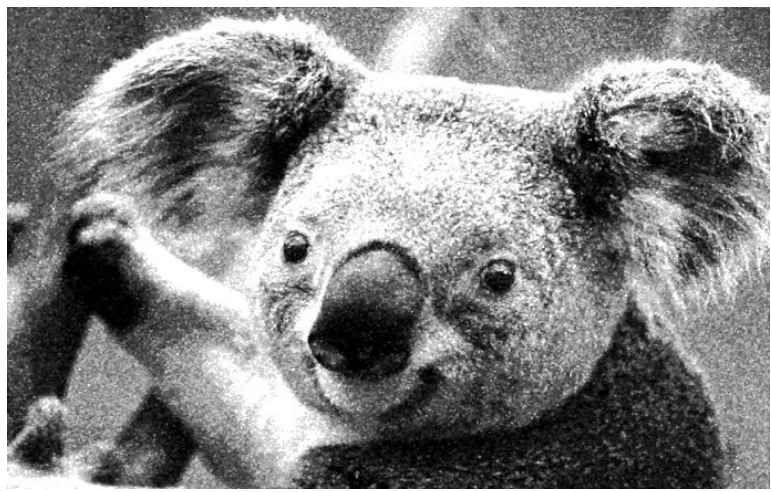

Fig 20: K_gauss_max_hist.gif
Table 2: Value of new quality index

\begin{tabular}{|c|c|c|}
\hline \multirow{2}{*}{$\begin{array}{c}\text { Noise/ } \\
\text { Technique }\end{array}$} & \multicolumn{2}{|c|}{ New Quality Index } \\
\cline { 2 - 3 } & After Max filter & $\begin{array}{c}\text { After Max filter } \\
\text { and Histogram } \\
\text { equalization }\end{array}$ \\
\hline Salt and pepper & 0.7841 & 0.8793 \\
\hline Speckle & 0.8182 & 0.9488 \\
\hline Gaussian & 0.7949 & 0.9625 \\
\hline
\end{tabular}

\section{CONCLUSION}

New quality index is by far the best quality parameter. The value of index should be near 1 in order to be a good value of index. The experiment shows that the value increases and becomes nearly equal to 1 , when the technique of histogram equalization is applied to order static filtered images. Now conclude from the said technique, Sequence of order static and histogram equalization give the better results for De-noising, this conclusion verified from new quality index parameter.

\section{REFERENCES}

[1] R.C. Gonzalez and R.E. Woods, Digital Image processing (3rd Edition).Upper Saddle River, NJ, USA: Prentice Hall, Inc 2006.

[2] mohammed ghouse, dr. m. siddappa, "adaptive techniques based high impulsive noise detection and reduction of a digital image," Journal of Theoretical and Applied Information Technology, (C) 2005 - 2011 JATIT \& LLS.

[3] Anmol Sharma, Jagroop Singh, "Image Denoising using Spatial domain filters: A Quantitative study," IEEE 6th International congress on image and signal processing.

[4] A. K. Jain, "Fundamentals of Digital Image Processing," Englewood Cliffs,NJ: Prentice-Hall, 1989.

[5] Poorna banerjee das gupta, "Analytical comparison of noise reduction filters for image restoration using SNR estimation," International journal of computer trends and technology (IJCTT) vol.17, no. 3, November 2014.

[6] Priyanka Kamboj, versha rani, "A brief study of various noise model and filtering technique," Journal of global research in computer science (JGRCS) Vol.4, no.4, april2013.

[7] Ravneet Kaur, Er. Navdeep Singh, "Image Restoration-A Survey," IOSR Journal of Computer Engineering (IOSRJCE) Volume 16, Issue 4, Ver.3 (Jul-Aug 2014) PP 107-111.

[8] Zhou wang, Alan c. bovik, "A universal image quality index," IEEE signal processing letters, Vol.XX, No. Y, march 2002.

[9] Archana, Sheenam, Amit Chhabra, "comprehensive review of Denoising techniques in image restoration," International Journal of Innovative Research in Computer and Communication Engineering (ijircce) Vol.2, Issue6, June2014. 
[10] Nishtha Attlas, Dr. Shefali Gupta, "Wavelet based Techniques for Speckle Noise Reduction in Ultrasound Images," International Journal of Engineering Research and Applications (ijera) vol. 4, Issue 2, February 2014, pp.508-513

[11] Anutam, rajni, "Performance Analysis of Image Denoising with Wavelet Thresholding methods for different levels of decomposition," The International Journal of Multimedia \& its Applications(IJMA) vol.6, no.3, June 2014

[12] Ajay kumar das, "Review on image Denoising techniques," International journal of emerging technology and advanced Engineering (ijetae) vol.4, issue 8, August 2014.

[13] Sarita Dangeti, "Denoising techniques- A comparison," Andhra university college of Engineering, may 2003.

[14] Agarwal Ankita, Kumar Tarun ' Various Image Denoising Performance Parameters: A Review', published in $2^{\text {nd }}$ international conference on VLSI communication and networks. (VCAN-2015) Organized by Institute of Engineering And Technology. North extn., M.I.A., Alwar-301030, India, 18-19, April 2015. 